Published in final edited form as:

Science. 2007 September 21; 317(5845): 1687.

\title{
The Future of Personal Genomics
}

\author{
Amy L. McGuire ${ }^{1}$, Mildred K. Cho ${ }^{2}$, Sean E. McGuire ${ }^{3}$, and Timothy Caulfield 4 \\ 1 Center for Medical Ethics and Health Policy, Baylor College of Medicine, Houston, TX 77030, USA
}

2 Stanford Center for Biomedical Ethics, Stanford University, Palo Alto, CA, USA

3 Division of Radiation Oncology, University of Texas MD Anderson Cancer Center, Houston, TX, USA

4 Health Law Institute, University of Alberta, Edmonton, AB, Canada

On 31 May 2007, James Watson was handed a miniature hard drive containing his personal genome sequence, which was subsequently uploaded onto publicly accessible databases. Craig Venter's personal genome was published a few months later (1). These projects represent research milestones. They also present an opportunity to examine the ethical, social, and clinical implications of personal genomics.

Excitement over these projects has been tremendous. Many are willing to pay a hefty price to be next. Scientists predict that within 5 years DNA sequencing technologies will be affordable enough that personal genomics will be integrated into routine clinical care (2). Companies are responding by offering their services for ancestry tracing, forensics, nutritional advice, reproductive assistance, and even social networking. It will not be long before companies are able to offer a "Face-book-like service centered around our genomes" (3). The medical community needs to consider the ways in which routine generation of this information will affect our health system and how this information might be used outside the medical context.

Despite limited treatment options for many genetic conditions, genetic testing has revolutionized the clinical management of patients for many disorders. Building on this history, companies and genetic testing laboratories have begun to develop technologies for genetic testing on a broader scale. For example, Baylor College of Medicine offers microarray analyses to evaluate a fetus for over 65 disorders or for genomic errors associated with autism or mental retardation (4).

We currently face an inflection point in clinical medicine as we move from specific diagnostic tests for particular disorders to much broader assays for variants whose effects we do not yet fully understand. In addition, the effects of any single gene on common diseases are generally small, and their interactions with environmental factors remain largely unknown. For example, despite enthusiasm about recent genomewide association studies that report an association between coronary heart disease and a common variant on chromosome 9 , the actual risk of heart disease was only increased from $1 \%$ to $1.6 \%$ in homozygotes $(5,6)$.

These studies are invaluable for understanding disease pathogenesis, but the present utility of this information for making treatment decisions is limited. Just because an association between genetic variation and disease is statistically significant does not mean that it is clinically meaningful (7). Moreover, simply knowing genetic risks and disease predispositions may not lead to better health decisions (8). For some, it might lead to fatalism and reduced compliance with healthy choices. As a result, many clinicians are "not at all enthusiastic about rushing out

*Author for correspondence. E-mail: amcguire@ bcm.edu.

Routine generation of whole-genome sequences will pose many health system challenges. 
to test people in the clinic" for these genes (7). Although the scientific value of genomic research has been enormous, these emerging technologies have only had marginal impact on health care to date, at least at the population level (9).

Some fear that personal genomes may become the genetic version of whole-body magnetic resonance imaging scans, which might lead to a population of "worried well" seeking followup investigations that could burden already-strained health-care systems. Physicians knowledgeable in genetics are the best guard against this concern. However, physicians are illprepared for current genetic testing information (10). The deluge of risk information from widespread use of whole-genome sequencing would greatly magnify this dilemma (11). Research and training for health-care providers is necessary for personal genomic information to affect patient care.

Only clinically meaningful genomic test results should be integrated into medical decisionmaking. Clinical practice guidelines should be developed, considering how best to use and present genomic information, and whether and how to withhold information that patients do not want to know. Failure to anticipate what may become standard of care could result in these issues being decided through malpractice litigation.

As personal genomics advances, questions about social justice and the cost of whole-genome sequencing will loom large, especially in countries (including the United States) where there is limited access to basic health-care services for many. Should private health insurance companies and public health systems pay for DNA sequencing, genetic analysis, counseling, and follow-up clinical care? (12). Will physicians be reimbursed for the additional time spent educating patients about the significance of genetic risk information? Payers will likely decline coverage for genomic testing and counseling until it can be associated with improved patient outcomes and quality of care (13).

The potential clinical application of genomic information is great, as exemplified by the recent U.S. Food and Drug Administration approval of a label change for warfarin to include information on how genetic variations may affect drug response (14). However, successful integration of personal genomics into routine clinical care will require clear standards, multidisciplinary collaboration, and careful consideration of the ethical, social, and clinical implications.

\section{References and Notes}

1. Levy, S., et al. PLoS Biology. 2007. p. 0001-0032.www.plosbiology.org

2. Wade, N. New York Times. 1 June. 2007 p.

A19

www.nytimes.com/2007/06/01/science/01 gene.html?ex=1183521600\&en=3ff34724cb1d6a0c\&ei=5070

3. Lei, H-H. Personal genome company 23andMe receives Google investment. 22 May. 2007 www.eyeondna.com/2007/05/22/personal-genome-company-23andme-receives-google-investment/

4. Chromosomal Microarray Analysis, Baylor College of Medicine, www.bcm.edu/cma/professional.htm.

5. McPherson R, et al. Science 2007;316:1488. [PubMed: 17478681]

6. Helgadottir A, et al. Science 2007;316:1491. [PubMed: 17478679]

7. Couzin J, Kaiser J. Science 2007;316:820. [PubMed: 17495150]

8. Carlsten C, Burke W. JAMA 2006;296:2480. [PubMed: 17119145]

9. Scheuner MT, Rotter JI. Genet Med 2006;8:141. [PubMed: 16540747]

10. Guttmacher AE, Porteous ME, McInerney JD. Nat Rev Genet 2007;8:151. [PubMed: 17230201]

11. Grosse SD, Khoury MJ. Genet Med 2006;8:448. [PubMed: 16845278]

12. Silversides A. Can Med Assoc J 2007;176:315. [PubMed: 17261824]

Science. Author manuscript; available in PMC 2008 January 30. 
13. Department of Health and Human Services (DHHS), Coverage and Reimbursement of Genetic Tests and Services: Report of the Secretary's Advisory Committee on Genetics, Health, and Society (February 2006); www4.od.nih.gov/oba/SACGHS/reports/CR_report.pdf.

14. Center for Drug Evaluation and Research, U.S. Food and Drug Administration, DHHS. New labeling information for warfarin (marketed as Coumadin). 16 August. 2007 www.fda.gov/cder/drug/infopage/warfarin/default.htm

15. We thank R. A. Gibbs for valuable contributions. Supported by the Greenwall Foundation, Baylor College of Medicine, Genome Alberta, and The Ethical, Legal, and Social Implications (ELSI) Research Program, National Human Genome Research Institute (NHGRI-ELSI), NIH, grants R01HG004333 and P50HG003389. 